\title{
LA GRAVITACION COMO UN MODELO DE CUERDA Y SU CUANTIZACION EN EL ESPACIO DE MINKOWSKI
}

\author{
Fulgencio Villegas Silva ${ }^{a}$ \\ ${ }^{a}$ Departamento de Física Atómica y Nuclear - Facultad de Ciencias Físicas \\ Universidad Nacional Mayor de San Marcos. Apartado Postal 14-0149, Lima 14, Perú.
}

\section{Resumen}

Se considera las ecuaciones de campo gravitacional de Einstein, identificándolas con las funciones que describen la inmersión del espacio-tiempo en un espacio plano encontrando así un modelo de teoría de campo. Se hace uso del Lagrangiano de Hilbert de tal manera que los campos dinámicos midan la curvatura del espaciotiempo donde el tensor energía-momento es proporcional al tensor de Einstein.

Palabras Claves: Gravitación, Teoría de campos, Cuerdas.

\begin{abstract}
The gravitational field equations of Einstein are considered, identifying them with the function that describe to the immersion of the space-time at a flat space finding a model of field theory. Hilbert's Lagrangian is used in such a way that dynamics fields measure the curvature of the space- time where the energy-momentum tensor is proportional to the Einstein's tensor.
\end{abstract}

Keywords: Gravitation, Field theory, Strings.

\section{Introducción}

La teoría de cuerdas es en la actualidad la principal alternativa para la superación de una serie de divergencias entre dos teorías físicas fundamentales, la mecánica cuántica y la relatividad general. Es también la única propuesta para la unificación de las cuatro fuerzas de interacción que conocemos: electromagnética, débil, fuerte y gravitacional.

Una teoría completa sobre la naturaleza debe incorporar la física clásica, la mecánica cuántica y la relatividad general. Esta teoría completa es la teoría de la gravedad cuántica. Los primeros intentos en tratar de conciliar la teoría de la gravedad de Einstein junto con la mecánica cuántica no tuvieron el éxito esperado. Los procedimientos de cálculos más sencillos que funcionan en el caso de la física de partículas daban respuestas infinitas que no tenían sentido alguno.

En la década de 1970 nació la teoría de cuerdas; aún cuando nació como un intento por entender la fuerza fuerte, fue obvio que esta teoría lo que realmente hacía era describir la gravedad y que podría ser utilizada para resolver la contradicción entre la mecánica cuántica y la gravedad. La longitud de Planck $\left(\sqrt{G / h c^{3}} \approx 10^{-33} \mathrm{~cm}\right)$ y el tiempo de Planck $\left(\sqrt{G / h c^{5}} \approx 5 \times 10^{-44} s\right)$ son las unidades naturales de un nivel de la realidad aún desconocido, muchísimo más pequeño que el mundo cuántico. En el mundo de Planck, la 
fuerza gravitacional vuelve a ser de fundamental importancia: los fenómenos cuánticos y gravitacionales se relacionan íntimamente entre sí y ni la mecánica cuántica ni la relatividad general son válidas por sí solas. Si el campo gravitacional es en realidad una curvatura del espacio-tiempo, debemos suponer que en el mundo de Planck, donde dominan los efectos cuánticos y gravitacionales, el espacio-tiempo posee fluctuaciones cuánticas como cualquier campo.

Prácticamente, desde que la mecánica cuántica tomó la forma con que se la conoce actualmente, muchos físicos intentaron crear una teoría cuántica de la gravitación. El intento más reciente tiene que ver con lo que se conoce como teoría de las supercuerdas. La manera en que las teorías normales son cuantizadas es partiendo de una configuración simple; en este caso podría ser un espacio-tiempo plano y luego considerar pequeñas desviaciones o pequeñas excitaciones a su alrededor. En este trabajo se trata de introducir la gravitación como una teoría de campo en el espacio de Minkowski y así demostrar que es posible su cuantización en dicho espacio-tiempo.

\section{La Gravitación como una Teoría de Campo en el Espacio de Minkowski}

Considerando una variedad Riemaniana $V_{n}$ de ndimensiones con métrica $\mathrm{g}_{\mathrm{ab}}(\mathrm{x}), \mathrm{y}$ un espacio plano $E_{N}$ de $\mathrm{N}$-dimensiones con métrica $\mathrm{G}_{\mathrm{AB}}(\mathrm{x})$; con coordenadas locales $\mathrm{x}^{\mathrm{a}}, \mathrm{a}=0, \ldots, \mathrm{n}-1$ y $\mathrm{x}^{\mathrm{A}}, \mathrm{A}=0, \ldots, \mathrm{N}-1$, en $V_{n}$ y $\mathrm{E}_{\mathrm{N}}$ respectivamente. De acuerdo con el teorema de inmersión local [3] la variedad Riemaniana $V_{n}$ se puede sumergir isométricamente en $\mathrm{E}_{\mathrm{n}}$. La inmersión está dada por:

$$
X^{A}=X^{A}\left(X^{a}\right)
$$

con rango

$$
\left(X_{a}^{A}\right)=n, \quad \mathrm{X}_{a}^{A}=\partial_{a} X^{A}
$$

Ambas métricas se relacionan por:

$$
g_{a b}=G_{A B}{ }_{a}^{A} X{ }_{b}^{B}
$$

Donde la condición de metricidad esta dada por

$\nabla_{\lambda} g_{a b}=0$, escrito como:

$$
G_{A B}=\mathrm{Y}_{a b}^{A} X_{\lambda}^{B}=0
$$

Donde $Y_{a b}^{A}$ son los tensores de Gauss definidos por:

$$
\mathrm{Y}_{a b}^{A}=X \underset{a ; b}{A}+\bar{\Gamma}_{B C}^{A} X_{a}^{B} X_{b}^{c}
$$

siendo $\bar{\Gamma}_{B C}^{A}$ los símbolos de Christoffel de $E_{N}$ restringidos a $\mathrm{V}_{\mathrm{n}}$. El escalar de curvatura esta dado por:

$$
\begin{gathered}
R=G^{a b \lambda \rho}{ }_{A B}{ }_{a b}^{A}{ }^{B}{ }_{\lambda \rho}^{B} \\
G^{a b \lambda \rho}=g_{g}^{a b}{ }_{g}^{\lambda \rho}-\frac{1}{2}\left(g^{a \lambda}{ }_{g}^{b \rho}+a_{g} a \rho^{b \lambda}\right) \\
M_{A B}=\eta_{A B}-X_{A \lambda}^{\lambda \rho_{X}}
\end{gathered}
$$

$$
\text { Con } X_{A \lambda}=\eta_{A B} X_{\lambda}^{B}, \quad X_{a b}^{A}=\partial_{a} \partial_{b} X^{A}
$$

Considerando el lagrangiano de Hilbert $L=R \sqrt{|g|}$, como una funcional de las coordenadas $X^{A}$. Tenemos que las ecuaciones de campo son [4]:

$$
G^{a b}{ }_{a b}^{A}=0
$$

Donde $\mathrm{G}^{\mathrm{ab}}$ es el tensor de Einstein. Por tanto el tensor de momento-energía esta dado por:

$$
\mathrm{T}_{b}^{a}(L)=X{ }_{b}^{A} \pi_{A}^{a}(L)+X_{b \lambda}^{A} N_{A}^{\lambda a}(L)-\delta_{b}^{a} L=0
$$

donde $\pi_{A}^{a}(L)$ y $N_{A}^{a b}(L)$ son los momentos generalizados que define el lagrangiano de Hilbert y que están dados como [5] 


$$
\begin{gathered}
\pi_{A}^{a}(L)=\frac{\partial L}{\partial X_{a}^{A}}-d_{b}\left[\frac{\partial L}{\partial X_{a b}^{A}}\right]=-2 \sqrt{-g} a^{a \lambda}{ }_{A \lambda} \\
N_{A}^{a b}(L)=\frac{\partial L}{\partial X_{a b}^{A}}=2 \sqrt{-g} M_{A B}^{G}{ }^{a b c d_{X}}{ }_{c d}^{B}
\end{gathered}
$$

Las coordenadas $\mathrm{X}^{\mathrm{A}}$ juegan el rol de campos, mientras que $\mathrm{X}^{\mathrm{a}}$ son coordenadas en el espacio base. Dando a los campos una dependencia explícita de las coordenadas $\mathrm{X}^{\mathrm{a}}$, esto significa fijar un calibre. Sean $\mathrm{X}^{\mathrm{A}}=\left(\mathrm{Y}^{\mathrm{a}}, \phi^{\alpha}\right)$ las coordenadas en $\mathrm{E}_{\mathrm{N}}$ con $\alpha=1, \ldots, \mathrm{N}-\mathrm{n}$. $\mathrm{V}_{\mathrm{n}}$ se define dando $\mathrm{X}^{\mathrm{A}}$ como funciones de $\mathrm{n}$ parámetros $\mathrm{X}^{\mathrm{a}}$, las coordenadas en $\mathrm{E}_{\mathrm{n}}$ como aquellas en $\mathrm{V}_{\mathrm{n}}$, es decir $\mathrm{Y}^{\mathrm{a}}=\mathrm{X}^{\mathrm{a}}$. Las coordenadas $\phi$ son los campos dinámicos. Entonces la métrica en $\mathrm{V}_{\mathrm{n}}$ toma la forma:

$$
g_{a b}^{*}=\hat{g}_{a b}+\eta_{\alpha \theta}{ }_{a}^{\alpha} \phi_{b}^{\theta}
$$

donde $\phi_{a}^{\alpha}=\partial{ }_{a}{ }^{\alpha} ; \hat{\mathrm{g}}_{a b}$ es la métrica de $\mathrm{M}_{\mathrm{n}}$. Las funciones $\phi$ miden la diferencia con la geometría de fondo $M_{n}$ del espacio $V_{n}$. Físicamente, los campos $\phi$ miden la curvatura del espacio-tiempo. Considerando que $\mathbf{M}_{n}$ es un espacio de Minkowski, entonces este modelo es una teoría de campo en un espacio de Minkowski. De este modo el espacio-tiempo de Minkowski aparece como el espacio base sobre el cual se debe formular la teoría de campo para el campo gravitacional. Donde las ecuaciones de campo se reducen a:

$$
\begin{gathered}
G^{a b}\left(\Gamma_{a b}^{\lambda}-\hat{\Gamma}_{a b}^{\lambda}\right)=0 \\
G_{\phi}^{a b}{ }_{a ; b}^{\alpha}=0
\end{gathered}
$$

Que son las ecuaciones que fijan el calibre y la dinámica respectivamente, siendo el respectivo lagrangiano:

$$
L^{*}=\sqrt{-g} G^{a b \lambda \rho}{ }_{\alpha \theta{ }_{a b}{ }^{\alpha} \lambda{ }_{\lambda \rho}^{\theta}}
$$

donde $\quad M_{\alpha \theta}=\eta_{\alpha \theta}-\phi_{\alpha \lambda}{ }^{\lambda \rho} \phi_{\theta \rho}$

Siendo ahora el tensor de momento-energía:

$$
\mathrm{T}_{b}^{a}(L)=2 \sqrt{-g} \hat{g}_{a \sigma} G{ }^{\sigma a}
$$

De tal manera que el tensor completamente contravariante y simétrico es:

$$
\mathrm{T}^{a b}(L)=\hat{g}^{a \lambda} \mathrm{T}_{\lambda}^{a}(L)=2 \sqrt{-g} G^{a b}
$$

\section{Conclusiones}

Se ha elaborado un modelo de teoría de campo en un espacio-tiempo de Minkowski, siendo éste el espacio base sobre el cual se debe formular la teoría de campo para el campo gravitacional. Lo cual indica que la cuantización del campo gravitacional es posible, dado que se reduce a la cuantización de campo en un espacio de Minkowski.

\section{Referencias}

[1] Green, J.H. Schwarz and E. Witten, Superstring Theory, Cambridge University Press (1987).

Polchinski, String theory. Vol. 2, Cambridge Press.

[2] Cupatitzio Ramirez, Conformal field theories in 2 dimensiones and string theory. Revista Mexicana de Física Vol. 39 (1993).

[3] E. Witten, string theory dynamics in various dimebnsions, Nucl. Phys. B443 85, hepth/9503124 (1995).

[4] L.P: Eisenhart, Riemannian Geometry, Princeton University Press (1926).

[5] T. Regge and C. Teitelboim, Proceedings of the First Marcel Grossman Meeting, Trieste, Italy, 1975, de. R. Ruffini (Amsterdam, NorthHolland (1977).

[6] M. Ferraris and M. Francaviglia, J. Math. Phys. 26. 1243 (1985). 